\title{
ANÁLISIS COMPARATIVO DE PROCESOS HISTÓRICOS DURANTE EL PERÍODO DE INTEGRACIÓN REGIONAL EN VALLES DE LA PROVINCIA DE LA RIOJA (ARGENTINA)
}

\author{
COMPARATIVE ANALYSIS OF HISTORICAL PROCESSES DURING \\ THE PERIOD OF REGIONAL INTEGRATION IN THE VALLEYS \\ OF LA RIOJA PROVINCE (ARGENTINA)
}

\author{
Adriana B. Callegari ${ }^{1}$ y María E. Gonaldi ${ }^{2}$
}

\begin{abstract}
Las investigaciones actuales sobre la problemática Aguada están indicando que, a pesar de compartir un substrato común, existieron marcadas diferencias regionales, producto de procesos culturales e históricos divergentes. Es dentro de esta línea de investigación que realizamos un análisis comparativo entre sociedades Aguada con registros arqueológicos que evidencian situaciones históricas diferenciadas. Los ámbitos considerados corresponden a dos microrregiones de los sectores centrales de los valles de Vinchina y Antinaco, provincia de La Rioja, Argentina, comprendidas en el sector meridional de La Aguada (González 1977 y 1998). Las principales diferencias se materializan en el patrón de instalación-asentamiento y en la elección, dentro del repertorio iconográfico Aguada, de diferentes motivos para la decoración cerámica. En el valle de Vinchina se buscó una estrategia de invisibilidad y en la decoración cerámica predominan los motivos geométricos. En el Valle de Antinaco prevalece una estrategia de alta visibilidad, evidenciada en la construcción de espacios públicos de carácter monumental, y, en la decoración cerámica, los motivos figurativos de fuerte contenido simbólico.

Con el objetivo de enmarcar temporalmente los sistemas de sitios, en este trabajo profundizamos en el análisis de los fechados radiocarbónicos obtenidos, cuyos resultados evidenciaron procesos sociales diacrónicos entre ambas microrregiones.

Palabras claves: Aguada meridional, modelos temporales.

Present research related to La Aguada archaeology shows that, despite sharing a common background, strong regional differences stemming from divergent cultural and historical processes existed. A comparative analysis was undertaken between two Aguada societies from two micro-regions of the central section of the Valleys of Vinchina and Antinaco. The valleys mentioned are both included in the southern sector of La Aguada, La Rioja Province, Argentina (González 1977, 1998). The main differences are found in the installation-establishment pattern, and the choice, within the La Aguada iconographic repertoire, of different patterns of ceramic decoration. In the Valley of Vinchina an invisibility strategy was developed and in ceramic decorations geometric patterns predominate. In the Valley of Antinaco a strategy of high visibility prevailed, as shown in the building of public spaces of a monumental nature, and in the ceramic decoration of figurative patterns with a strong symbolic content.

With the aim of identifying a time frame for the Aguada sites, we analyzed radiocarbon dating results, which demonstrate diachronic social processes between both micro-regions.
\end{abstract}

Key words: Aguada southern sector, temporal models.

Según González $(1977,1998)$ las sociedades que habitaron la región valliserrana del noroeste argentino (NOA) durante el período Medio (550900 d.C.), identificada y denominada por el autor como "Cultura de la Aguada" (González 1961-64), compartieron una ideología religiosa materializada en un repertorio iconográfico con un alto contenido simbólico, donde el felino, representado tan- to de forma realista como abstracta, tuvo un rol relevante. No obstante, dataciones cronológicas absolutas, realizadas recientemente tanto por el mencionado autor como por otros investigadores que trabajan con la problemática Aguada en diferentes ámbitos, han ampliado su rango temporal (Baldini et al. 2002; Boschín et al. 1999; Callegari 1997-1999; González 1998; Gordillo 1997-1999 y

1 Sección Arqueología - Instituto de Ciencias Antropológicas, Facultad de Filosofía y Letras, Universidad de Buenos Aires, 25 de Mayo 217. Tercer Piso, 1002 Buenos Aires. rincon3@ speedy.com.ar

2 Departamento de Antropología y Arqueología, Museo de Ciencias Naturales, Universidad Nacional de La Rioja, Av. Laprida y R. Favaloro, 5300 La Rioja. megonaldi@arnet.com.ar 
2005). Asimismo están indicando que, a pesar de compartir un substrato común, existieron diferencias regionales y microrregionales producto de procesos histórico-culturales diferenciados. En líneas generales, entre el 900 y 1.100 de la era en gran parte de la región valliserrana se van desvaneciendo los indicadores Aguada que caracterizan al período Medio o de Integración Regional.

Entre el repertorio de motivos decorativos que caracterizan a Aguada figuran personajes con tocados y pieles de jaguar que portan estandartes, hachas, cabezas-trofeos; motivos antropozoomorfos; manchas; garras e imágenes fantásticas que, en algunos casos, presentan más de una lectura, etc. Todos ellos fueron plasmados en distintos tipos de soportes, como son la alfarería, miniaturas de hueso, tallas en piedra, metalurgia y en el arte rupestre. Cabe destacar la alta calidad alcanzada en la fabricación de cerámicas cocidas a altas temperaturas, tanto en atmósferas oxidantes como reductoras. En la metalurgia, elaborada con cobres arsenicales, se logró un gran refinamiento, especialmente en la fabricación de objetos suntuarios, como son las hachas y los discos que en algunos casos fueron ricamente decorados.

González (1977, 1998) advierte sobre la variabilidad espacial de Aguada y propone tres sectores con características propias, pero con una simbología en común, a los cuales denomina sector oriental, sector septentrional y sector meridional. Este último comprende el norte de la provincia de San Juan y el norte y centro de la provincia de $\mathrm{La}$ Rioja, que incluye la zona que nos ocupa.

En líneas generales, la cultura material del sector meridional se caracteriza por la presencia de cerámicas cocidas en atmósferas oxidantes con decoración pintada con motivos zoomorfos, como el cóndor, mulita, aves y de tipo abstracto, además de los motivos anteriormente mencionados. Las figurillas humanas de arcilla o talladas en piedra con complicados peinados son típicas de este sector (González 1977). Otros investigadores sostienen que en el transcurso de dicho período tuvo lugar un proceso de unificación social e ideológico, proponiendo la denominación de período de Integración Regional en lugar de período Medio, como más representativo del proceso de integración entre diferentes sociedades y tradiciones culturales preexistentes (Núñez Regueiro y Tartusi 1987, 1996-1997; Pérez Gollán y Heredia 1987). Estos autores concuerdan que con Aguada se instaura una organización sociopolítica a nivel de jefaturas, convalidada por una fuerte ideología religiosa. Por nuestra parte, planteamos que en algunos ámbitos de la región valliserrana -como es la microrregión del sector central del valle de Vinchina- parte de la ideología religiosa Aguada, el sistema simbólicoiconográfico asociado y muchos de los rasgos característicos de la cultura material habrían continuado vigentes durante el período de los Desarrollos Regionales.

\section{Los Sistemas de Sitios}

Las microrregiones que aquí nos ocupan, donde se radican los proyectos de investigación en curso $^{1}$, se ubican en los sectores centrales de los valles de Vinchina y Antinaco, separados por el Cordón de Famatina (Figura 1). Se encuadran dentro de la variedad de climas cálidos de sierras y bolsones, en la región fitogeográfica de monte occidental y en el distrito zoogeográfico subandino (Lafón 1970). Los registros arqueológicos recuperados en ambas microrregiones, aunque con rasgos diferenciados, se adscriben a la entidad Aguada.

\section{Microrregión del sector central del valle de Vinchina}

Los faldeos terminales de la ladera occidental del cordón de Famatina y cerro Aspercito forman profundas entradas en forma de U conocidas localmente como "rincones". La amplia visión que se tiene desde sus laderas del ambiente del fondo de valle, los endicamientos y vertientes naturales de agua, debido a la gran permeabilidad de los suelos (Turner 1964), la protección que brindó de los vientos e insolación, etc., habrían favorecido su elección como lugar de instalación por la sociedad Aguada local, construyendo un sistema de sitios en su interior (descripción detallada de los rincones y el sistema de sitios en Callegari 2004).

La presencia de diferentes tipos de estructuras a nivel intra e intersitio ${ }^{2}$, además de indicar una jerarquización entre ellos, responderían a las funciones preponderantes que habrían desempeñado, distinguiéndose tres grupos de asentamientos:

(1) Asentamientos Residenciales: Comprende los sitios (a) Rincón del Toro (de Aparicio 194042 y Callegari 2001) y (b) la Fortaleza del Cerro el Toro (de la Fuente 1971). 


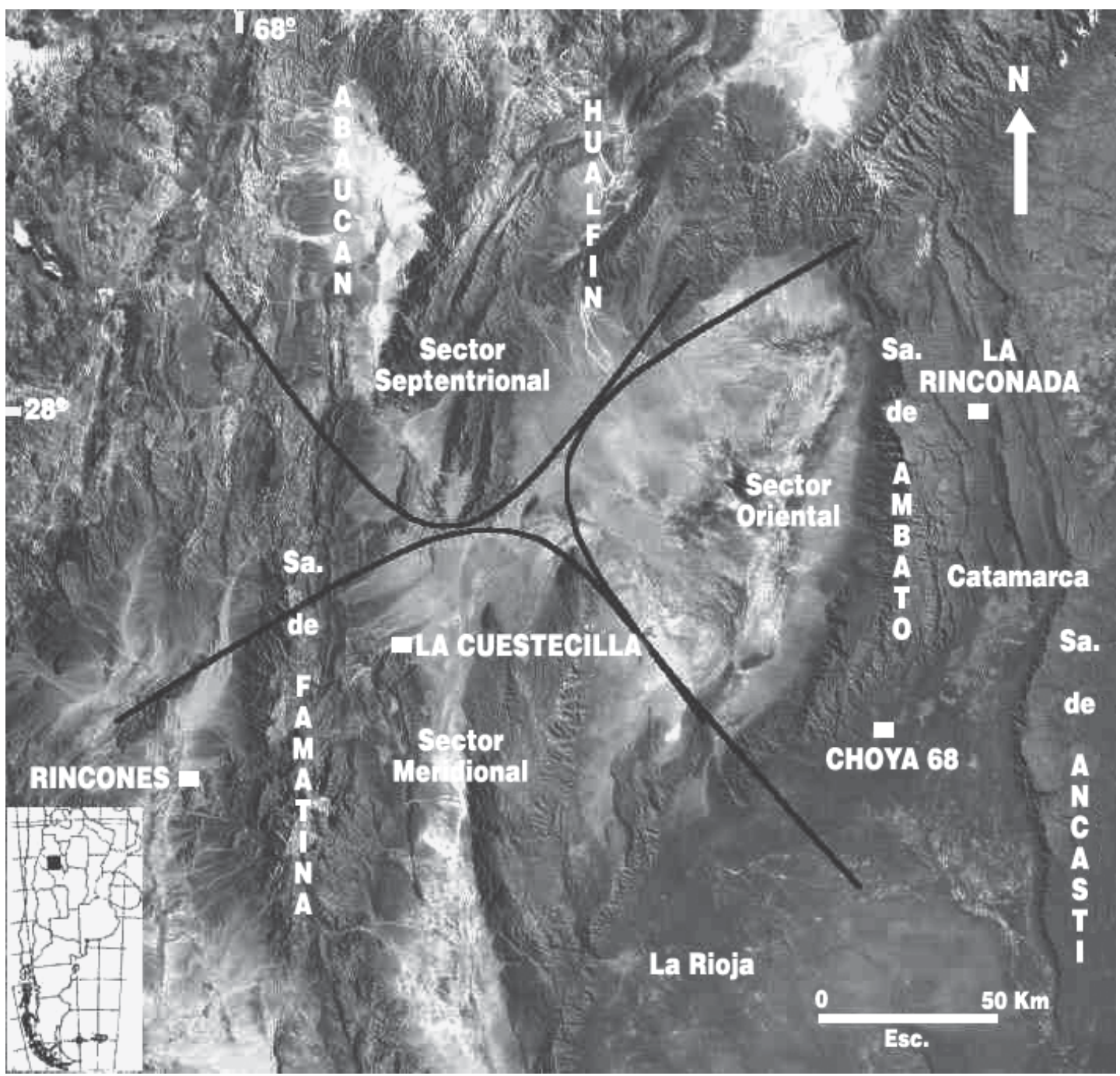

Figura 1. Ubicación de las microrregiones en los valles de La Rioja, Argentina. Location of the micro-regions in the valleys of La Rioja Province, Argentina.

(a) El sitio Rincón del Toro está compuesto por 52 unidades domésticas, simples y compuestas, que se escalonan sobre dos de las laderas del cerro el Toro, ocho plataformas en las partes más elevadas y con mejor visión del valle y cinco muros de contención. Sobre 13 rocas planas distribuidas entre los recintos se identificaron una serie de grabados, algunos de ellos con los típicos motivos de la iconografía Aguada.

(b) La Fortaleza del Cerro el Toro se ubica sobre la cima del cerro homónimo, desde donde se tiene una amplia visión y control del valle de Vinchina. La instalación está compuesta por 50 unidades domésticas de diferentes tipos y se encuentra rodeada por precipicios, salvo el sector septentrional. Presenta una única vía de acceso por el faldeo sur, que está controlada en su tramo superior por dos estaciones de vigilancia con arte rupestre en sus inmediaciones. En este grupo de asentamientos residenciales se incluyó al sitio El Carmen ubicado en el ambiente fondo de valle. Presenta, además del material cerámico Aguada, los tipos Sanagasta y Negro s/Rojo adscribibles al período de los Desarrollos Regionales.

(2) Asentamientos Productores: Compuesto por los rincones La Cantera, El Corral, Las Trojitas y Peña Rosada. Su funcionalidad se infiere por la presencia de canchones, andenes de 
cultivo y corrales resguardados por grandes murallas.

(3) Asentamientos de Control: integrado por los rincones Adga, de Caballos, Adoquines y La Puntilla. Un rasgo compartido por todos los sitios fue la elección de una estrategia de invisibilidad (Criado Boado 1999) en cuanto al emplazamiento en el interior de los rincones. Asimismo, al utilizar las rocas graníticas de los cerros en las construcciones lograron mimetizarse con el paisaje. Todas las estructuras de tipo defensivas y de control están orientadas hacia el fondo del valle, donde se ubican el sitio El Carmen y 48 concentraciones de materiales arqueológicos.

Entre el material cerámico Aguada recuperado en los Rincones predominan los tipos pintados, decorados con motivos geométricos y excepcionalmente figurativos. También están presentes los grupos cerámicos Ordinarios y Allpatauca.

El tamaño de la población para cada uno de los sitios se calculó a partir del número de unidades domésticas u otra clase de estructura que pudo haber funcionado de esa manera. Se tomaron en consideración también las superficies del o los recintos que habrían sido usados como habitación (Hassan 1978) ${ }^{3}$. Se estimó de esta manera una población media -entre el máximo y el mínimo- de 480 habitantes para el ambiente de los rincones (Figura 2). Dado el emplazamiento estratégico de la Fortaleza en la cima del Cerro el Toro, las construcciones de tipo defensivo-ofensivas que presenta y el tamaño prácticamente igual al del sitio Rincón del Toro (rangos 1 y 2), planteamos que ambos sitios habrían funcionado en estrecha vinculación. La Fortaleza habría cumplido la función de albergar y defender a la población que habitó en los rincones en caso de ataque, especialmente la del Rincón del Toro, por tal motivo no se la incluyó en el cálculo de la población.

A partir de los promedios obtenidos para cada sitio, se analizó el sistema bajo la norma rango/ tamaño $(r / t)$, cuya representación bilogarítmica ${ }^{4}$ se representa en la Figura 3.

La relación entre el rango de los asentamientos y su tamaño se basa en el supuesto que el rango más alto de la jerarquía (r) está en relación con los diferentes niveles de toma de decisiones y con el grado de integración alcanzado. Siendo, además, una herramienta sensible para medir la emergen-

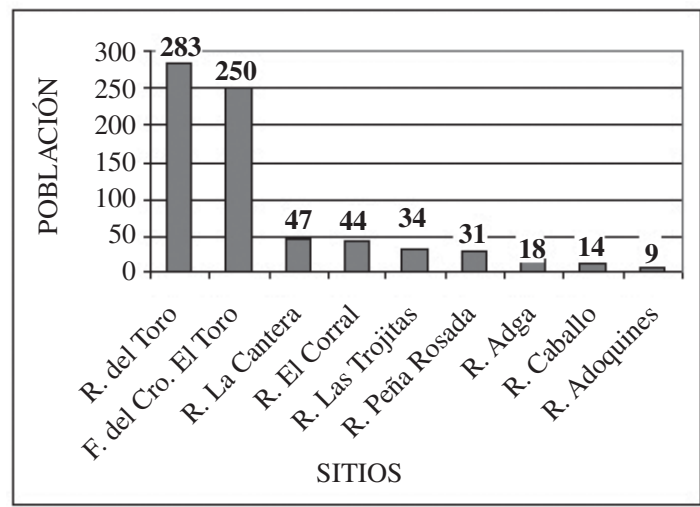

Figura 2. Población estimada para la microrregión central del valle de Vinchina.

Estimated population for the central micro-regions of the Vinchina Valley.

cia del control de las funciones asociadas con los procesos de complejidad social (Johnson 1977; Payter 1983).

\section{Marco temporal para la microrregión del sector central del valle de Vinchina}

Todas las mediciones radiocarbónicas fueron realizadas en distintos momentos en el Laboratorio de Tritio y Radiocarbono (LATyR) de la Facultad de Ciencias Naturales y Museo de la Universidad de La Plata, salvo una que se dató en el Center to Applied Isotoped. Studies University of Georgia (UGA). En la Tabla 1 se consignan las 16 dataciones $\mathrm{C}^{14}$ obtenidas para el sector central del valle de Vinchina, para su calibración se utilizó el programa CALIB Rev. 5.0.1 (Stuiver et al 2005)

Dado que el rango de edades calibradas por el método de intersección con la curva "A" (que permite convertir una edad $\mathrm{C}^{14}$ en años calendarios, Figini 1999) es algo amplio para el estudio de los procesos sociales del período que nos ocupa, en la calibración también se seleccionó el método de área de probabilidad "B"6, obteniéndose de esta manera una cronología más acotada. Es así que, en las desviaciones estándares correspondientes a 1 y 2 sigmas de las edades calibradas de la Tabla 1, se seleccionaron aquellas que presentaban el porcentaje más alto en el área de probabilidad. Asimismo, para obtener una cronología más precisa, a las edades calibradas se les aplicaron procedimientos estadísticos.

En la Figura 4 se expresan de forma comparativa todos los fechados calibrados por el método A 


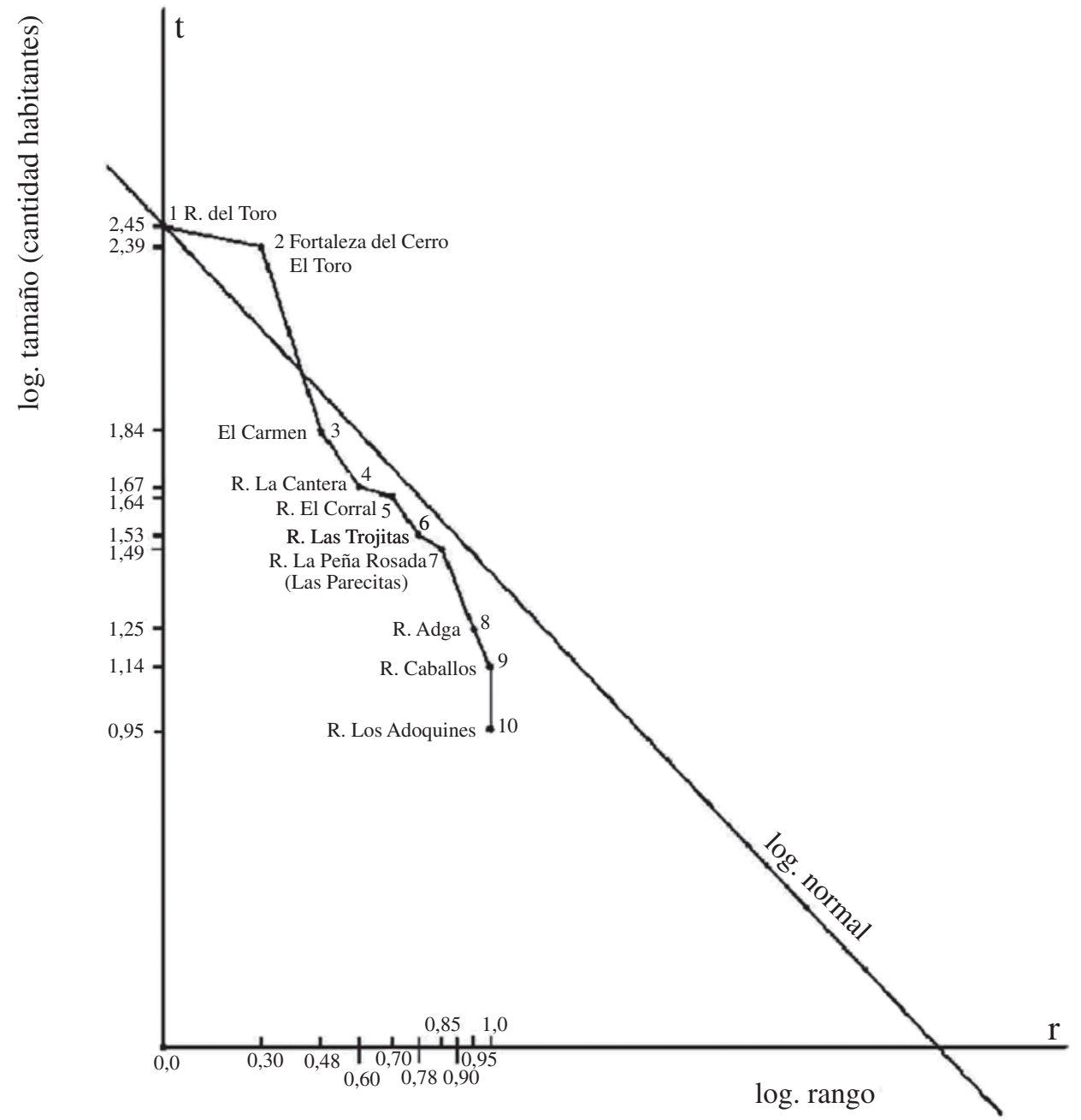

Figura 3. Representación del rango/tamaño del sistema de sitios del sector central del valle de Vinchina.

Representation of the range/size of the site systems for the central sector of the Vinchina Valley.

de intersección con la curva que, según su distribución en el gráfico, estarían indicando un proceso. Para contrastar dicha hipótesis se aplicó el test estadístico T (tiempo) ${ }^{7}$ a la serie de fechados $\mathrm{C}^{14}$, con excepción de los dos fechados más modernos (LP 1017 y LP 798), ya que se separaban notoriamente de la serie.

El valor T obtenido para las 14 muestras resultó mayor que el de chi ${ }^{2}$ n- 1 grado de libertad y $5 \%$ de significación obtenido de las tablas estadísticas, que indicó que las edades radiocarbónicas comparadas son significativamente diferentes (todos valores ob- tenidos para $\mathrm{T}$ y chi $^{2} \mathrm{n}-1$ grado de libertad y $5 \%$ de significación se consignan en la Tabla 2).

El paso siguiente fue calcular los estadísticos T agrupando los fechados, según donde se producían los quiebres más notorios en el gráfico de la Figura 4, comenzando por los tres fechados $\mathrm{C}^{14}$ con dataciones más antiguas. En los análisis estadísticos no se consideraron los fechados LP 1292, 809 y 196, pues en el gráfico se ubican en las zonas de transición de los quiebres que fueron tomados como referencia para dividir los grupos de fechados. 
Tabla 1. Fechados radiocarbónicos y calibraciones del sector central del valle de Vinchina. Radiocarbon dates and calibrations of the central sector of the Vinchina Valley.

\begin{tabular}{|c|c|c|c|c|c|}
\hline Sitio & Código & Procedencia & $\begin{array}{c}\text { Edad } \\
\text { radiocarbónica } \\
\text { a.p. }\end{array}$ & cal. 1 sigma & cal. 2 sigma \\
\hline \multirow{5}{*}{$\begin{array}{l}\text { Rincón del } \\
\text { Toro }\end{array}$} & LP 919 & $\begin{array}{l}\text { Rec. 2-Nivel } 3 \\
\text { Carbón de fogón }\end{array}$ & $700 \pm 60$ & $\begin{array}{c}1.263-1.315 \text { d.C. } \\
687-635 \text { a.p. }\end{array}$ & $\begin{array}{c}1.220-1.332 \text { d.C. } \\
730-618 \text { a.p. }\end{array}$ \\
\hline & LP 885 & $\begin{array}{l}\text { Rec. 1-Nivel } 3 \\
\text { Carbón de fogón }\end{array}$ & $680 \pm 50$ & $\begin{array}{c}1.280-1.315 \text { d.C. } \\
670-635 \text { a.p. }\end{array}$ & $\begin{array}{c}\text { 1260-1333 d.C. } \\
\text { 690-617 a.p. }\end{array}$ \\
\hline & LP 1017 & $\begin{array}{l}\text { Rec. 3-Nivel } 3 \\
\text { Carbón disperso }\end{array}$ & $270 \pm 50$ & $\begin{array}{c}1.520-1.589 \text { d.C. } \\
430-361 \text { a.p. }\end{array}$ & $\begin{array}{c}1.480-1.678 \text { d.C. } \\
470-272 \text { a.p. }\end{array}$ \\
\hline & LP 1186 & $\begin{array}{l}\text { Rec. 19-Nivel } 6 \\
\text { Carbón de fogón }\end{array}$ & $530 \pm 60$ & $\begin{array}{c}1.390-1.440 \text { d.C. } \\
560-510 \text { a.p. }\end{array}$ & $\begin{array}{l}1.376-1.455 \text { d.C. } \\
574-495 \text { a.p. }\end{array}$ \\
\hline & LP 1292 & $\begin{array}{l}\text { Rec.19-Nivel } 7 \\
\text { Carbón de fogón }\end{array}$ & $740 \pm 70$ & $\begin{array}{l}1.215-1.303 \text { d.C. } \\
735-647 \text { a.p. }\end{array}$ & $\begin{array}{l}1.159-1.333 \text { d.C. } \\
791-617 \text { a.p. }\end{array}$ \\
\hline \multirow{2}{*}{$\begin{array}{l}\text { Fortaleza del cerro } \\
\qquad \text { El Toro }\end{array}$} & LP 1031 & $\begin{array}{l}\text { Rec. 25-Sondeo I } \\
\text { Carbón disperso }\end{array}$ & $680 \pm 70$ & $\begin{array}{c}1.277-1.326 \text { d.C. } \\
673-624 \text { a.p. }\end{array}$ & $\begin{array}{c}1.235-1.407 \text { d.C. } \\
715-543 \text { a.p. }\end{array}$ \\
\hline & LP 1282 & $\begin{array}{l}\text { Rec. 20-Sondeo II } \\
\text { Carbón disperso }\end{array}$ & $1.030 \pm 50$ & $\begin{array}{l}\text { 962-1.037 d.C. } \\
\text { 988-913 a.p. }\end{array}$ & $\begin{array}{l}\text { 893-1.063 d.C. } \\
\text { 1057-887 a.p. }\end{array}$ \\
\hline R. Las Trojitas & LP 1183 & $\begin{array}{l}\text { Sondeo I } \\
\text { Carbón disperso }\end{array}$ & $620 \pm 40$ & $\begin{array}{l}1.301-1.328 \text { d.C. } \\
\text { 649-622 a.p. }\end{array}$ & $\begin{array}{c}1.295-1.404 \text { d.C. } \\
\text { 655-546 a.p. }\end{array}$ \\
\hline R. La Cantera & LP 1276 & $\begin{array}{l}\text { Sondeo I } \\
\text { Carbón disperso }\end{array}$ & $590 \pm 50$ & $\begin{array}{c}1.305-1.355 \text { d.C. } \\
645-595 \text { a.p. }\end{array}$ & $\begin{array}{c}\text { 1.297-1.422 d.C. } \\
\text { 653-528 a.p. }\end{array}$ \\
\hline $\begin{array}{l}\text { El Carmen } \\
\text { Rec. } N^{\circ} 5\end{array}$ & LP 196 & $\begin{array}{l}\text { Cuad. II-Nivel } 6 \\
\text { Carbón piso- } \\
\text { disperso }\end{array}$ & $740 \pm 40$ & $\begin{array}{l}1.255-1.296 \text { d.C. } \\
695-654 \text { a.p. }\end{array}$ & $\begin{array}{l}1.216-1.303 \text { d.C. } \\
734-648 \text { a.p. }\end{array}$ \\
\hline \multirow{6}{*}{$\begin{array}{c}\text { El Carmen } \\
\text { Estructura de } \\
\text { descarte M II }\end{array}$} & LP 773 & $\begin{array}{l}\text { Cuad. 5-Sect.12 } \\
\text { Carbón } \\
\text { concentrado }\end{array}$ & $995 \pm 50$ & $\begin{array}{l}\text { 987-1.044 d.C. } \\
\text { 963-906 a.p. }\end{array}$ & $\begin{array}{l}962-1.163 \text { d.C. } \\
\text { 988-787 a.p. }\end{array}$ \\
\hline & LP 477 & $\begin{array}{l}\text { Sondeo 1-Sect. f } \\
\text { Carbón } \\
\text { concentrado }\end{array}$ & $930 \pm 80$ & $\begin{array}{l}\text { 1.024-1.164 d.C. } \\
\text { 926-786 a.p. }\end{array}$ & $\begin{array}{l}\text { 983-1.261 d.C. } \\
\text { 967-689 a.p. }\end{array}$ \\
\hline & LP 452 & $\begin{array}{l}\text { Sondeo 1-Sect. k } \\
\text { Carbón } \\
\text { concentrado }\end{array}$ & $610 \pm 80$ & $\begin{array}{l}1.335-1.373 \text { d.C. } \\
615-577 \text { a.p. }\end{array}$ & $\begin{array}{c}1.270-1.441 \text { d.C. } \\
\text { 680-509 a.p. }\end{array}$ \\
\hline & LP 809 & $\begin{array}{l}\text { Cuad. 6-Sect. } 4 \\
\text { Maíz }\end{array}$ & $470 \pm 60$ & $\begin{array}{c}1.402-1.482 \text { d.C. } \\
548-468 \text { a.p. }\end{array}$ & $\begin{array}{l}1.388-1.524 \text { d.C. } \\
562-426 \text { a.p. }\end{array}$ \\
\hline & LP 798 & $\begin{array}{l}\text { Cuad. 4-Sect. } 4 \\
\text { Carbón } \\
\text { concentrado }\end{array}$ & $250 \pm 50$ & $\begin{array}{c}1.627-1.676 \text { d.C. } \\
323-274 \text { a.p. }\end{array}$ & $\begin{array}{c}1.607-1.685 \text { d.C. } \\
\text { 343-265 a.p. }\end{array}$ \\
\hline & UGA 762 & $\begin{array}{l}\text { Cuad. 6-Sect. } 3 \\
\text { Fragmento cestería }\end{array}$ & $570 \pm 50$ & $\begin{array}{c}1.310-1.354 \text { d.C. } \\
\text { 640-596 a.p. }\end{array}$ & $\begin{array}{c}1.377-1.432 \text { d.C. } \\
573-518 \text { a.p. }\end{array}$ \\
\hline
\end{tabular}

LP: LATyR. Laboratorio de Tritio y Radiocarbono, Facultad de Ciencias Naturales y Museo, UNLP, La Plata. UGA: Center to Applied Isotope. Studies University of Georgia.

Los sigmas fueron calculados con el método B de área relativa bajo la distribución de probabilidades. 


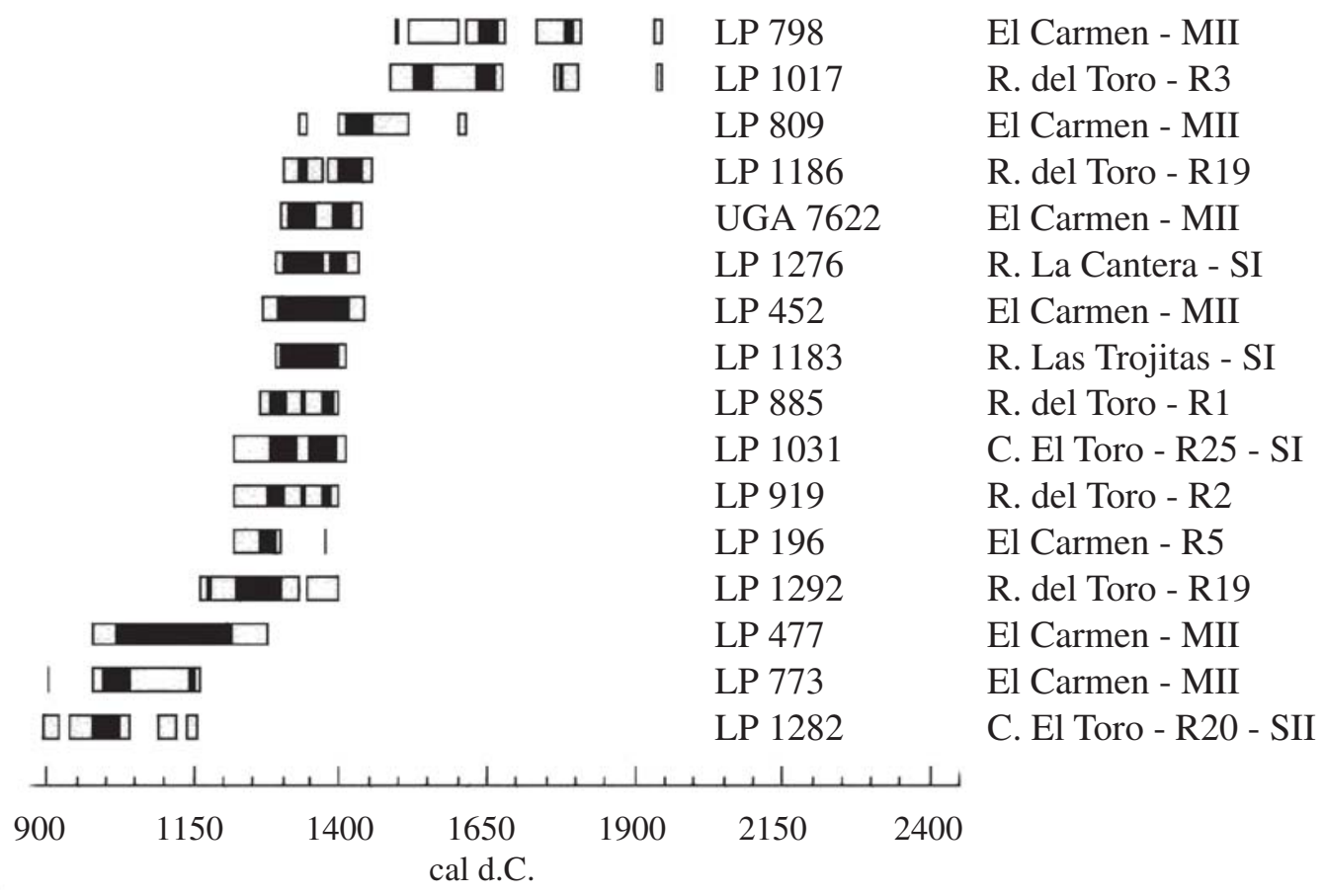

Figura 4. Fechados radiocarbónicos calibrados del sector central del valle de Vinchina. Calibrated radiocarbon datings for the central sector of the Vinchina Valley.

Tabla 2. Sector central del valle de Vinchina. Grupo A. Estadístico T. Central sector of the Vinchina Valley. Group A. T-test.

\begin{tabular}{cllcr}
\hline Código de la Muestra & \multicolumn{1}{c}{ Procedencia } & Material fechado & Fechado C ${ }^{14}$ a.p. & Error S \\
\hline LP 1282 & F. del C. El Toro - R 20 - SII & Carbón disperso & $1.030 \pm 50$ & 78,10 \\
LP 773 & El Carmen - MII-C5- Sect. 12 & Carbón concentrado & $995 \pm 50$ & 78,10 \\
LP 477 & El Carmen - MII - S1- Sect. F & Carbón concentrado & $930 \pm 80$ & 100,00 \\
\hline
\end{tabular}

\section{1) Grupo A (Tabla 3)}

El valor estadístico $\mathrm{T}$ indicó que las fechas comparadas corresponden a una misma edad verdadera, siendo la Mp y su error la mejor estimación de la edad real: $993 \pm 48$ a.p.

Los porcentajes más altos dentro del área de probabilidad son ${ }^{8}$ :

959-906 cal. a.p./991-1.044 cal. d.C. $(p=0,580-1 \sigma)$. 975-787 cal. a.p./975-1.163 cal. d.C. $(p=0,976-2 \sigma)$.
2) Grupo B (Tabla 4)

Para este grupo de dataciones el valor $\mathrm{T}$ indicó que las fechas comparadas estadísticamente corresponden a una misma edad verdadera, siendo la Mp la mejor estimación de la edad verdadera: $621 \pm 29$ a.p.

Los porcentajes más altos dentro del área de probabilidad son:

648-623 cal. a.p./1.302-1.327 cal. d.C. $(p=0,417-1 \sigma)$. 612-551 cal. a.p./1.338-1.399 cal. d.C. $(p=0,619-2 \sigma)$. 


\section{3) Grupo C (Tabla 5)}

La resultante de T marcó que las fechas comparadas estadísticamente corresponden a una misma edad verdadera, siendo la Mp la mejor estimación de la edad: $260 \pm 55$ a.p.
Los porcentajes más altos dentro del área de probabilidad son:

430-359 cal. a.p./ 1.520-1.591 cal. d.C. $(p=0,435-1 \sigma)$.

472-265 cal. a.p./ $1.478-1.685$ cal. d.C. $(p=0,762-2 \sigma)$.

Tabla 3. Sector central del valle de Vinchina. Grupo B. Estadístico T.

Central sector of the Vinchina Valley. Group B. T-test.

\begin{tabular}{llllr}
\hline Código de la Muestra & Procedencia & Material fechado & Fechado C ${ }^{14}$ a.p. & Error S \\
\hline LP 919 & R. del Toro - R2 - N3 & Carbón de fogón & $700 \pm 60$ & 84,85 \\
LP 885 & R. del Toro - R1 - N3 & Carbón de fogón & $680 \pm 50$ & 78,10 \\
LP 1031 & F. del C. el Toro & Carbón disperso & $680 \pm 70$ & 92,19 \\
LP 1183 & R. Las Trojitas - SI & Carbón disperso & $620 \pm 40$ & 72,11 \\
LP 452 & El Carmen M II - S 1- Sect. K & Carbón concentrado & $610 \pm 80$ & 100,00 \\
LP 1276 & R. La Cantera - SI & Carbón disperso & $590 \pm 50$ & 78,10 \\
UGA 7622 & El Carmen - C.6 - Sect. 3 & Fragmento de cestería & $570 \pm 50$ & 78,10 \\
LP 1186 & R. del Toro - R19 - N6 & Carbón de fogón & $530 \pm 60$ & 84,85
\end{tabular}

Tabla 4: Sector central del valle de Vinchina. Grupo C. Estadístico T. Central sector of Vinchina Valley. Group C. T-test.

\begin{tabular}{cllcr}
\hline Código de la Muestra & Procedencia & Material fechado & Fechado C ${ }^{14}$ a.p. & Error S \\
\hline LP 1017 & R. del Toro - R3 - N3 & Carbón disperso & $270 \pm 50$ & 78,10 \\
LP 798 & El Carmen - MII - C4 - Sect. 4 & Carbón concentrado & $250 \pm 50$ & 78,10
\end{tabular}

Tabla 5: Sector central del valle de Vinchina. Valores obtenidos en los cálculos del estadístico T y de chi $^{2}$ para n-1 grado de libertad y $5 \%$ significación.

Central sector of Vinchina Valley. Values obtained in the calculations of $T$-test and $c h i^{2}$ for $n=1$ degree of freedom and a $5 \%$ significance level.

Fechados analizados $\begin{gathered}\text { Número de } \\ \text { muestras } \\ (\mathrm{n})\end{gathered} \quad \mathrm{T} \quad$ chi $^{2}$

\begin{tabular}{lrrr}
$\begin{array}{l}\text { Toda la serie } \\
\text { (menos LP 1017 y 798) }\end{array}$ & 14 & 56,09 & 25,18 \\
Grupo A & 3 & 0,62 & 5,99 \\
Grupo B & 8 & 3,59 & 14,07 \\
Grupo C & 2 & 0,032 & 3,84 \\
\hline
\end{tabular}

\section{Microrregión del sector central del valle de Antinaco}

Los sitios individualizados en esta microrregión son La Cuestecilla, Cerrito Negro y El Cantadero. Todos ellos se emplazan en un ambiente de fondo de valle amplio y abierto (para una descripción más detallada de los sitios consultar Raviña y Callegari 1988 y Callegari et al. 2000).

En el sitio La Cuestecilla, el más grande y de mayor complejidad estructural, hemos realizado trabajos de prospección, recolecciones de superficie, relevamientos planimétricos y excavaciones. En los otros sitios únicamente llevamos a cabo prospecciones y recolecciones de material cerámico de superficie, que nos permitieron ubicarlos temporalmente en el período de Integración o Medio, y adscribirlos culturalmente a la entidad Aguada. En 
la decoración de la cerámica Aguada pintada predominan los motivos figurativos de una alta carga simbólica con representaciones felínicas y también los de tipo abstracto.

Dado que aún no hicimos la planimetría de la totalidad de los sitios, no pudimos realizar cálculos estimativos del tamaño de la población y, en consecuencia, aún no fue posible rankearlos según la norma $\mathrm{r} / \mathrm{t}$, como lo hiciéramos con los sitios del sector central del valle de Vinchina. No obstante, la diferencia notoria entre sus tamaños y la complejidad estructural intrasitio observadas, avalarían la existencia de una jerarquización entre ellos.

Los sitios se ubican en un ambiente de fondo de valle primando una estrategia de visibilidad e inclusive de monumentalización intrasitio (Criado Boado 1999), como es específicamente el caso de La Cuestecilla, con la presencia de espacios públicos con construcciones monumentales (plataformas y montículo) y algunos espacios domésticos arquitectónicamente jerarquizados con relación a otros (Callegari et al. 2000). Otro rasgo que lo diferencia del caso anterior es la ausencia de construcciones de carácter defensivo, hecho que nos lleva a postular que sus habitantes no se encontraban presionados por situaciones de conflicto.

\section{Marco temporal para la microrregión del sector central del valle de Antinaco}

Las investigaciones que hemos desarrollado en el valle se encuentran en una etapa preliminar con respecto al caso anterior. Motivo por el cual las muestras de fechados $\mathrm{C}^{14}$ con que contamos para esta microrregión provienen de un sólo sitio, $\mathrm{La}$ Cuestecilla, mientras que para la microrregión considerada del valle de Vinchina fueron tomadas de diferentes sitios. En el análisis de los fechados de La Cuestecilla se siguió el mismo procedimiento que para la microrregión anterior.

En la Tabla 6 se presentan las edades $\mathrm{C}^{14}$ obtenidas de las excavaciones realizadas en La Cuestecilla, y en la Figura 5 se grafican las edades calibradas por el método A de intersección con la curva. El estadístico T aplicado a la totalidad de la serie indicó que las 6 edades radiocarbónicas comparadas son significativamente diferentes (todos valores obtenidos para $\mathrm{T}$ y de $\mathrm{chi}^{2} \mathrm{n}-1$ grado de libertad y $5 \%$ de significación se consignan en la Tabla 7).

Las fechas también fueron agrupadas según donde se observan los quiebres en el gráfico. A los grupos obtenidos se les aplicó el estadístico T, comenzando por las cuatro muestras con dataciones más antiguas.

Tabla 6. Fechados radiocarbónicos y calibraciones del sitio La Cuestecilla (Sector central del valle de Antinaco). Radiocarbon dates and calibrations of La Cuestecilla site (Central sector of Antinaco Valley).

\begin{tabular}{|c|c|c|c|c|}
\hline Código & Procedencia & $\begin{array}{c}\text { Edad } \\
\text { radiocarbónica } \\
\text { a.p. }\end{array}$ & cal. 1 sigma & cal. 2 sigmas \\
\hline LP 922 & $\begin{array}{l}\text { G6 - Recinto1-N2 } \\
\text { Carbón concentrado }\end{array}$ & $1.020 \pm 50$ & $\begin{array}{l}976-1040 \text { d.C. } \\
974-910 \text { a.p. }\end{array}$ & $\begin{array}{l}940-1071 \text { d.C. } \\
1.010-879 \text { a.p. }\end{array}$ \\
\hline LP 936 & $\begin{array}{l}\text { G6-Recinto1-N3 } \\
\text { Carbón de fogón }\end{array}$ & $1.260 \pm 50$ & $\begin{array}{l}\text { 685-782 d.C. } \\
1.265-1.168 \text { a.p. }\end{array}$ & $\begin{array}{l}\text { 667-785 d.C. } \\
1.283-1.165 \text { a.p. }\end{array}$ \\
\hline LP 933 & $\begin{array}{l}\text { G6 - Recinto 1-N4 } \\
\text { Carbón. Debajo del piso asociado a enterratorio }\end{array}$ & $1.320 \pm 60$ & $\begin{array}{l}\text { 657-725 d.C. } \\
1.293-1.225 \text { a.p. }\end{array}$ & $\begin{array}{l}\text { 636-783 d.C. } \\
1.314-1.167 \text { a.p. }\end{array}$ \\
\hline LP 1034 & $\begin{array}{l}\text { G } 15-\mathrm{N} 2 \text { y } 3 \\
\text { Carbón disperso }\end{array}$ & $1.370 \pm 50$ & $\begin{array}{l}\text { 636-691 d.C. } \\
1.314-1.259 \text { a.p. }\end{array}$ & $\begin{array}{l}597-735 \text { d.C. } \\
1.353-1.215 \text { a.p. }\end{array}$ \\
\hline LP 909 & $\begin{array}{l}\text { G10 -Plataforma-N } 2 \text { y } 3 \\
\text { Carbón disperso }\end{array}$ & $1.150 \pm 60$ & $\begin{array}{l}\text { 909-976 d.C. } \\
\text { 1.041-974 a.p. }\end{array}$ & $\begin{array}{l}\text { 769-1.002 d.C. } \\
1.181-948 \text { a.p. }\end{array}$ \\
\hline LP 1189 & $\begin{array}{l}\text { Montículo } \\
\text { Carbón concentrado }\end{array}$ & $790 \pm 70$ & $\begin{array}{l}\text { 1.186-1.282 d.C. } \\
\text { 764-662 a.p. }\end{array}$ & $\begin{array}{l}1.150-1.303 \text { d.C. } \\
800-647 \text { a.p. }\end{array}$ \\
\hline
\end{tabular}




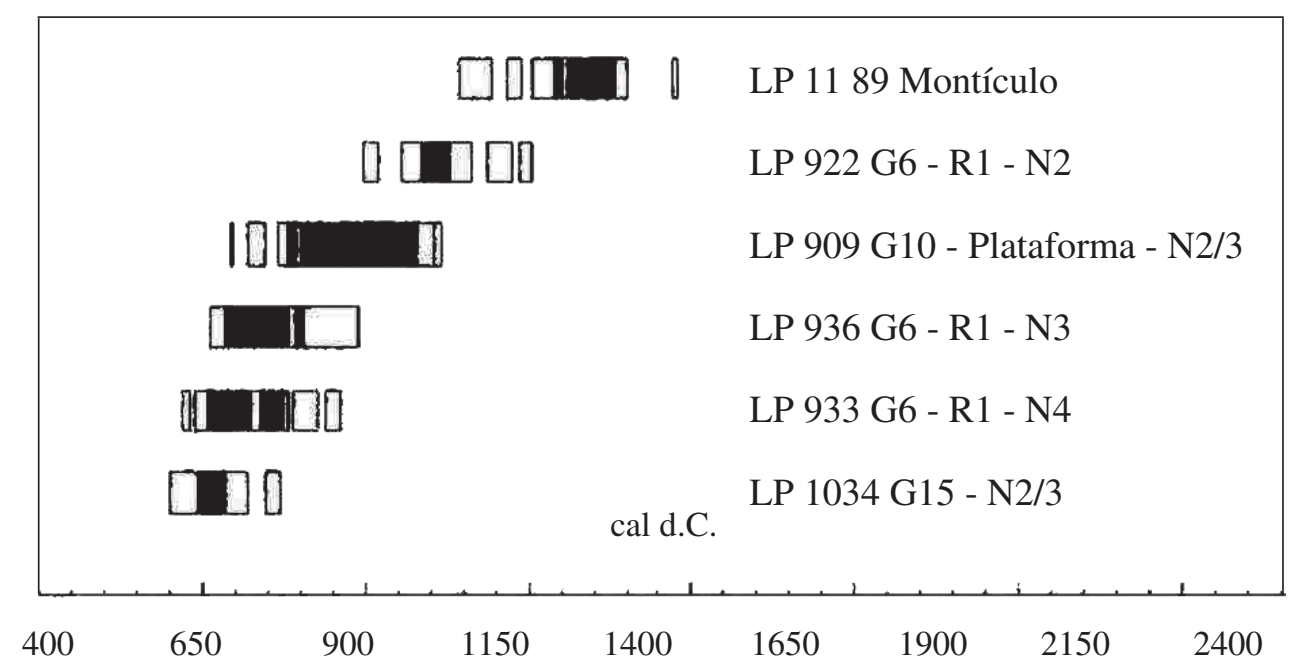

Figura 5. Fechados radiocarbónicos calibrados del sitio La Cuestecilla (sector central del valle de Antinaco). Calibrated radiocarbon dates for the La Cuestecilla site, in the central sector of the Antinaco Valley.

Tabla 7. Sitio La Cuestecilla (sector central del valle de Antinaco). Grupo A. Estadístico T. La Cuestecilla site (Central sector of Antinaco Valley). Group A. T-test.

\begin{tabular}{|c|c|c|c|c|}
\hline Código de la Muestra & Procedencia & Material fechado & Fechado $\mathrm{C}^{14}$ a.p. & Error $\mathrm{S}$ \\
\hline LP 1034 & L.C.- G15 - N 2 y 3 & Carbón disperso & $1.370 \pm 50$ & 78,10 \\
\hline LP 933 & L.C.- G6 - R1- N 4 & $\begin{array}{l}\text { Carbón. Debajo del piso } \\
\text { asociado a enterratorio }\end{array}$ & $1.320 \pm 60$ & 84,85 \\
\hline LP 936 & L.C.- G6 - R1 - N 3 & Carbón de fogón & $1.260 \pm 50$ & 78,10 \\
\hline LP 909 & L.C.- G10 - Plat.- N 2 y 3 & Carbón disperso & $1.150 \pm 60$ & 84,85 \\
\hline
\end{tabular}

Tabla 8. Sitio La Cuestecilla (sector central del valle de Antinaco). Grupo B. Estadístico T. La Cuestecilla site (Central sector of Antinaco Valley). Group B. T-test.

\begin{tabular}{cllcc}
\hline Código de la Muestra & Procedencia & Material fechado & Fechado C ${ }^{14}$ a.p. & Error S \\
\hline LP 922 & L.C.- G6 - R 1-N 2 & Carbón concentrado & $1.020 \pm 50$ & 78,10 \\
LP 1189 & L.C.- Montículo & Carbón concentrado & $790 \pm 60$ & 92,19 \\
\hline
\end{tabular}

\section{1) Grupo A (Tabla 8)}

El valor de T para este grupo de edades indicó que las mismas corresponderían a una misma edad verdadera, siendo Mp la mejor estimación de esa edad: $1.278 \pm 40$ a.p.

Los porcentajes más altos dentro del área de probabilidad son:
1.262-1.216 cal. a.p./688-734 cal. d.C. $(p=0,530-1 \sigma)$. $1.289-1.167$ cal. a.p. $/ 661-783$ cal. d.C. $(p=0,879-2 \sigma)$.

2) Grupo B (Tabla 9)

El T aplicado a este grupo señaló que las muestras pertenecen a una misma edad verdadera, siendo la Mp la mejor estimación de esa edad: $924 \pm 59$ a.p. 
Los porcentajes más altos dentro del área de probabilidad son:

917-788 cal. a.p./1.033-1.162 cal. d.C. $(p=1,000-1 \sigma)$. 934-727 cal. a.p./1.016-1.223 cal. d.C. $(p=0,983-2 \sigma)$.

Tabla 9. Sitio La Cuestecilla (sector central del valle de Antinaco). Valores obtenidos en los cálculos del estadístico $\mathrm{T}$ y de $\mathrm{chi}^{2}$ para $\mathrm{n}-1$ grado de libertad y $5 \%$ significación.

La Cuestecilla site (Central sector of Antinaco Valley). Values obtained in calculations of T-test and $c h i^{2}$ for $n=1$ degree of freedom and $a$ $5 \%$ significance level.

\begin{tabular}{lcrr}
\hline Fechados analizados & $\begin{array}{c}\text { Número de } \\
\text { muestras (n) }\end{array}$ & T & chi $^{2}$ \\
\hline Toda la serie & 6 & 31,72 & 11,98 \\
Grupo A & 4 & 3,96 & 7,68 \\
Grupo B & 2 & 3,62 & 3,84 \\
\hline
\end{tabular}

\section{Discusión y Conclusiones}

Las investigaciones que en los últimos años se vienen desarrollando con relación a la problemática Aguada, en general, y su cronología absoluta, en particular, han puesto de manifiesto la diversidad de procesos entre los diferentes ámbitos y aun entre diferentes valles. Algunos de los investigadores que han calibrado y aplicado procedimientos estadísticos a los fechados ya existentes así lo han manifestado en sus análisis (Bonnin y Laguens 1996; Gordillo 1997-1999).

Como se desprende de la Figura 6, las dos microrregiones con componentes Aguada aquí comparadas muestran un distanciamiento temporal en su desarrollo cultural, mostrando cada una un desarrollo temporal prolongado. Los fechados más tardíos del sitio La Cuestecilla son contemporáneos (se traslapan en el gráfico) con los más tempranos de los sitios ubicados en el ambiente de los rincones, y con los del sitio El Carmen emplazado en el ambiente de fondo de valle.

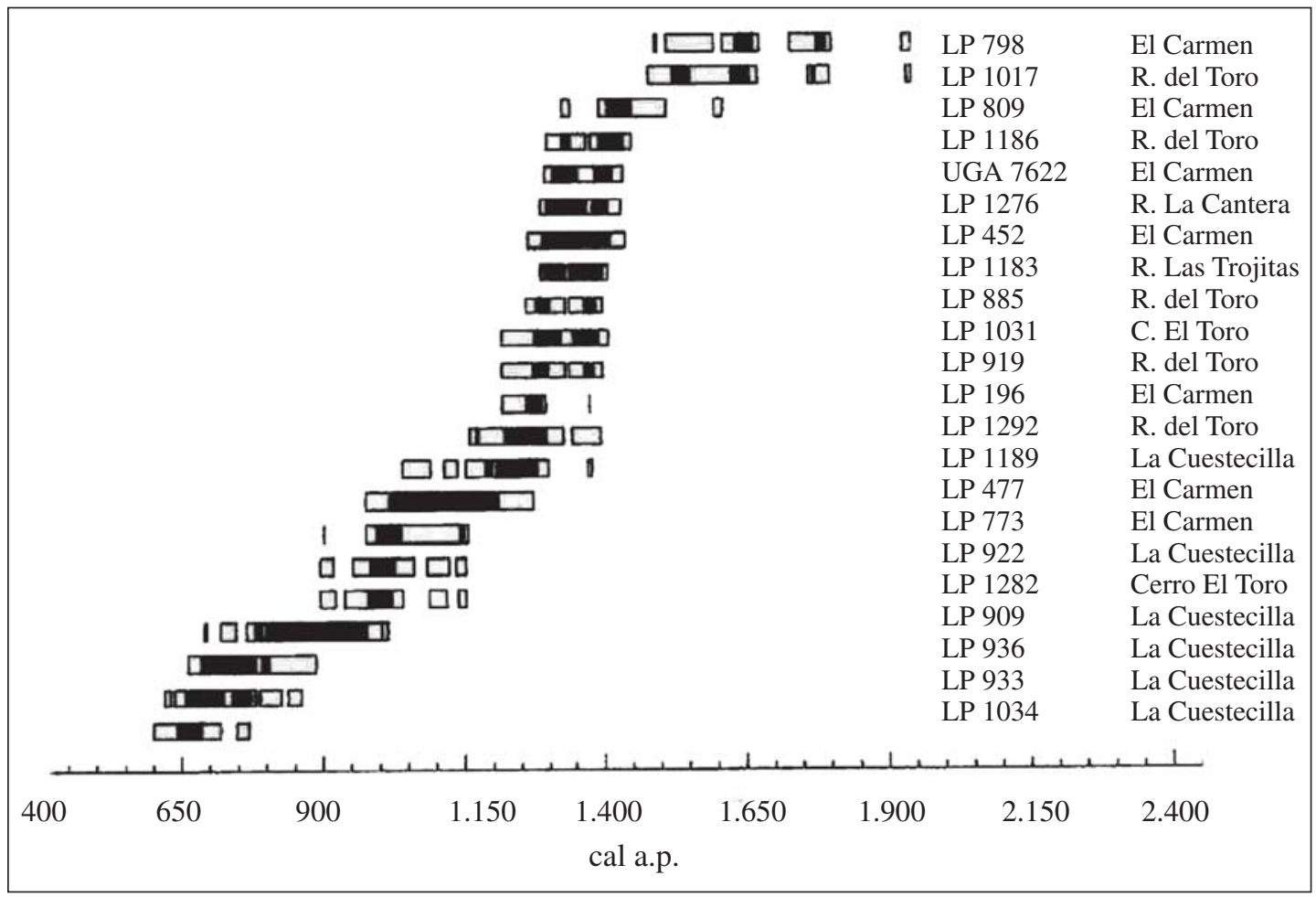

área negra: 1 sigma; área blanca: 1 y 2 sigmas.

Figura 6. Fechados radiocarbónicos calibrados para los sectores centrales del valle de Vinchina y valle de Antinaco (La Cuestecilla). Calibrated radiocarbon dates for the central sector of the Vinchina and Antinaco Valleys (La Cuestecilla). 
Los resultados de la calibración de los fechados $C^{14}(\operatorname{con} 2 \sigma)$ y las edades medias ponderadas obtenidas, señalan que:

(1) La Cuestecilla habría estado ocupada por aproximadamente 650 años (ca. 600-1.250 d.C.).

(2) Con relación a la microrregión estudiada del sector central del valle de Vinchina, el ambiente de fondo de valle habría estado ocupado por alrededor de 730 años (ca. 950-1.680 d.C.).

(3) El ambiente de los rincones habría sido habitado por un rango temporal menor, de aproximadamente 450 años (ca. 950-1.400 d.C.). Por el momento desestimamos la datación LP 1017 $\left(270 \pm 50\right.$ a.p. $\left.\mathrm{C}^{14}\right)$, debido a que la muestra consistente en carbón disperso en el piso de uno de los recintos del Rincón del Toro prácticamente no apareció asociada a ningún tipo de registro. Además, éste, como el resto de los sitios que componen el sistema, únicamente presentaron un único componente Aguada.

(4) Los datos obtenidos estarían indicando que la ocupación Aguada para el sector central del valle de Vinchina se habría prolongado por más tiempo que en otros ámbitos, iniciándose ca. 900-950 d.C. Esta fecha tradicionalmente marcaba el final de estas sociedades.

$\mathrm{Al}$ respecto, es importante señalar que dataciones recientes (Boschín et al. 1999; Baldini et al. 2002; Gordillo 2005) están mostrando la continuación de Aguada más allá del 1.000 de la era. Boschín y Llamazares han datado las pictografías Aguada de la sierra de Ancasti, con fechados que se distribuyen entre ca. 700 y 1.300 d.C. $(2 \sigma)$ (Boschín et al. 1999). González y su equipo obtuvieron fechados para Aguada provenientes de Choya 68 en el Depto. Capayán, Catamarca, que se distribuyen alrededor del 1.000 d.C. (Baldini et al. 2002). Gordillo, por su parte, ha informado para el sitio La Rinconada (Ambato, Catamarca) fechados que alcanzan 1.019-1.214 (LP 1206) y 1.036-1.283 (LP 932) cal. d.C. (con 2 $\sigma$ ) (Gordillo 2005).

El análisis que realizáramos al sistema de los rincones con la norma $\mathrm{r} / \mathrm{t}$ muestra un comienzo de complejidad y diferenciación sociopolítica, manifestado en un patrón de asentamiento con dos sitios de gran tamaño (rangos 1 y 2) que incluyen manifestaciones de arte rupestre. Bastante más abajo, en la escala espacial se distribuye el resto de los sitios de tamaño pequeño. Si se excluyese de la representación $r / t$ a La Fortaleza, ya que habría sido ocupada por los habitantes del Rincón del Toro y de los otros rincones en situaciones de conflicto, todos los sitios se distribuirían por debajo de la logarítmica normal, registrándose una marcada diferencia entre el rango 1 y el 3, hecho que estaría señalando una situación incipiente de concentración de poder y toma de decisiones. Por otro lado, la ubicación en el rango 3 de El Carmen nos lleva a pensar que respondió a otro sistema, correspondiente al ambiente de fondo de valle ${ }^{9}$.

La presencia en el ambiente de los rincones de grandes emprendimientos colectivos, que implicaron el manejo de cantidad de mano de obra como son las murallas del Rincón Las Trojitas, puestos de control, plataformas ubicadas en las cotas altas y sendas que comunicaron a los rincones sin necesidad de descender al fondo del valle, evidencian una gran energía invertida en la construcción de estructuras de tipo defensivas, lo que indicaría que la población se encontraba bajo una situación de inseguridad y conflictos latentes. Cabe destacar que las construcciones mencionadas están orientadas hacia el oeste, como para defenderse de un enemigo que hostigaba desde el ambiente del fondo del valle, donde se ubican el sitio El Carmen y las 48 concentraciones de materiales cerámicos correspondientes a los grupos Ordinarios, Sanagasta y Negro s/ Rojo, además del grupo cerámico Aguada. Mientras que en los rincones el material cerámico recuperado corresponde exclusivamente a los grupos cerámicos Aguada, Ordinario y Allpatauca (este último siempre asociado con Aguada). Al respecto, no podemos dejar de considerar la posibilidad que el grupo Sanagasta y/o el Negro/Rojo fueran confeccionados por pueblos que habitaban en el fondo de valle y que periódicamente entraban en conflicto con la población Aguada que, como estrategia de defensa literalmente, se habría "arrinconado". Asimismo, la serie de fechados presentada y su calibración muestran que en gran parte de la secuencia los sitios ubicados en el ambiente de los Rincones y El Carmen en el ambiente de fondo de valle fueron contemporáneos (Tabla 1 y Figura 4).

Es interesante señalar que a partir del $1.000 \mathrm{de}$ la era hay evidencias en casi todo el NOA de conflictos intercomunitarios generalizados (Nielsen 2001; Raffino 1988; Tarragó 1999). Un proceso similar informa Nielsen para la Quebrada de Humahuaca (Provincia de Jujuy) para la fase Muyu- 
na-Calete (ca. 900 -1.280 d.C.), con una cronología en parte coincidente con la de los Rincones. Según el autor, los pobladores de esta fase habrían elegido las zonas altas y de gran visibilidad para instalarse, como una estrategia de defensa frente al aumento de situaciones de beligerancia en la Quebrada. Otros rasgos que coinciden con el caso de los Rincones son una alta densidad en la edificación, diferencias de tamaño entre las estructuras, presencia de corrales, etc. (Nielsen 1996).

A diferencia del valle de Vinchina, en el sitio La Cuestecilla con una serie de fechados más tempranos para Aguada, no se ha registrado una estrategia de invisibilidad en cuanto a su emplazamiento ni presencia de estructuras defensivas que indiquen que sus pobladores hubiesen estado expuestos a potenciales situaciones de conflicto. Por el contrario, emplearon una estrategia de monumentalidad en la construcción de sus espacios públicos. No obstante, en los ca. 650 años de ocupación se evidencia un cambio en las características formales de los espacios públicos, seguramente relacionado con una variación en el ritual que en ellos se habrían desarrollado (Callegari et al. 2000), y acorde con el cambio ideológico que tuvo lugar hacia fines del período de Integración e inicio de los Desarrollos Regionales en todo el noroeste argentino.

Agradecimientos: A los dos evaluadores anónimos que, con sus acertados comentarios, contribuyeron a mejorar el trabajo. No obstante, todos los conceptos aquí vertidos son única responsabilidad de las autoras.

\section{Referencias Citadas}

Baldini, M., J. Carbonari, G. Cieza, M.E. de Feo, M.F. del Castillo, A. Figini, A.R. González, R. Huarte y J. Togo

2002 Primer análisis de la cronología obtenida en el sitio Choya 68 (Depto. Capayán, Provincia de Catamarca, Argentina). Estudios Atacameños 24:71-82.

Bonnin, M. y A. Laguens

1996 Evaluación de series de fechados radiocarbónicos del valle de Ambato. Publicaciones Arqueología 48:65-101.

Boschín, M.T., R. Hedges y A.M. Llamazares

1999 La datación absoluta del arte rupestre de la Argentina. Ciencia Hoy 50:55-65.

Callegari, A.

1997-1999 La transición a los Desarrollos Regionales en el oeste riojano. Actas del XII Congreso Nacional de Arqueología Argentina. Tomo 2:75-81. Facultad de Ciencias Naturales y Museo, La Plata.

2001 Los grabados del Rincón del Toro, el paisaje y su relación con el sistema iconográfico Aguada. Boletín del Museo Chileno de Arte Precolombino 8:21-33.

2004 Las poblaciones precolombinas que habitaron el sector central del valle de Vinchina entre el 900/950 y 1600/ 1650 d.C. (Dto. General Lamadrid, Provincia de La Rioja, Argentina). Relaciones XXIX: 81-111.

Callegari, A., M.E. Gonaldi y M.G. Raviña

2000 Paisaje social y ceremonialismo en La Cuestecilla (Dto. de Famatina, La Rioja). Actas del XIV Congreso Nacional de Arqueología Chilena. Tomo 1:875-893.

Criado Boado, F.

1999 Criterios y convenciones en arqueología del paisaje. Del terreno al espacio: planeamientos y perspectivas para la Arqueología del Paisaje. Capa 6:1-81.

de Aparicio, F.

1940/42 La tambería del Rincón del Toro. Publicaciones del Museo Etnográfico 4:239-251. de la Fuente, N.

1971 La Fortaleza del Cerro El Toro. Revista del Instituto de Antropología de Córdoba 1:10-23.

Figini, A. J.

1999 Análisis de la calibración en años calendarios de las edades C-14. Corrección para el hemisferio sur. Actas XII Congreso Nacional de Arqueología Argentina. Tomo 2:349-352.

Figini, A., R. Huarte, G.J. Gómez, J. Carbonari y A.C. Zubiaga 1983 Control estadístico en la determinación radiocarbónica en el LATYR: Consideraciones. Publicaciones 1-66.

González, A.R.

1961-64 La Cultura de La Aguada del NO.A. Revista del Instituto de Antropología de Córdoba 2:2-21.

1977 Arte Precolombino de la Argentina. Introducción a su Historia Cultural. Filmediciones Valero, Buenos Aires. 1998 Cultura de La Aguada. Arqueología y Diseños. Arte Precolombino. Filmediciones Valero, Buenos Aires.

Gordillo, I.

1997-1999 Problemas cronológicos del período Medio en el noroeste argentino. Actas del XII Congreso Nacional de Arqueología Argentina. Tomo 2: 362-371.

2005 Dimensión temporal del sitio La Rinconada. Su interpretación y aportes a la historia del período Medio. En La Cultura de La Aguada y sus Expresiones Regionales. Editorial de la Universidad Nacional de La Rioja (EUDELAR).

Hassan, F.A.

1978 Demographic archaeology. Advances in Archaeological Method and Theory 1:49-103.

Johnson, G.

1977 Aspects of regional analysis in archaeology. Annual Reviews Anthropology 6:479-508.

Lafón, H.R.

1970 Manual de Historia y Geografía de La Rioja. Geografía. Tomo 2. Editorial Riojana, La Rioja. 
Nielsen, A.

1996 Demografía y cambio social en la Quebrada de Humahuaca (Jujuy, Argentina) 700-1535 d.C. Relaciones XXI: 307-355.

2001 Evolución social en Quebrada de Humahuaca (AD 700-1536). En Historia Argentina Prehispánica, editado por E. Berberian y A. Nielsen, Tomo I, pp. 171-249. Editorial Brujas, Córdoba.

Núñez Regueiro, V. y M. Tartusi

1987 Aproximación al estudio del área pedemontana de Sudamérica. Cuadernos del Instituto Nacional de Antropología 12:125-160.

1996-1997 Los Orígenes de Aguada. Shincal 6:45-59.

Pérez Gollán, J. A. y O. Heredia

1987 Hacia un replanteo de la cultura de La Aguada. Cuadernos del Instituto Nacional de Antropología 12:161-179.

Payter, R.

1983 Expanding the scope of settlement analysis. En $\mathrm{Ar}$ chaeological Hammers and Theories, editado por
J. Moore y A. Keene, pp. 244-275. Academic Press, New York.

Raviña, M. G. y A. Callegari

1988 Hallazgos arqueológicos en el Cantadero (Dto. de

Famatina Pcia. de La Rioja). Revista de Antropología 4:10-17.

Raffino, R.

1988 Poblaciones Indígenas de la Argentina, Tipográfica Editora, Argentina, Buenos Aires.

Stuiver, M., P. J. Reimer y R.W. Reimer

2005 CALIB 5.0. [WWW program and documentation].

Tarragó, M.

1999 Las sociedades del sudeste andino. En Historia General de América Latina, editado por UNESCO, Vol. 1, pp. 465-480. Editorial Trotta, Buenos Aires.

Turner, J.C.

1964 Descripción geológica de la hoja 15 C. Vinchina (Provincia de La Rioja). Boletín 100:1-81.

\section{Notas}

1 Los proyectos en curso son: UBAC y T F 169 y F079; PIP 0089/98 y PICT 12182.

2 Los diferentes tipos de estructuras identificadas son: unidades domésticas simples pequeñas y grandes; unidades domésticas compuestas por dos o tres recintos con diferente tipo de circulación; recintos de control; plataformas; murallas; corrales; muros de contención, andenes y canchones; caminos, y un sistema de túneles y cámaras dispuestos por debajo de la Fortaleza del Cerro el Toro.

3 Se excavó completamente por lo menos una unidad doméstica de cada tipo (simples y compuestas, integradas las últimas por dos o tres recintos), evidenciándose, en todos los casos, un único piso de ocupación, lo cual indicó que fueron ocupadas durante un solo evento. Con relación a las estimaciones demográficas, a las cifras absolutas obtenidas se les agregó un $15 \%$ más, por posibles sesgos en el registro de algunos recintos por encontrarse tapados a causa de los deslizamientos de tierra y piedra que ocurren en los cerros. Dado que los resultados obtenidos en la calibración de los fechados marcaron un proceso, se consideró que el $70 \%$ de las unidades domésticas fueron habitadas contemporáneamente (Hassan 1978).

4 Debido a que en el sitio La Puntilla no se identificó ningún tipo de unidad doméstica, sino exclusivamente es- tructuras de carácter defensivas-ofensivas, no aparece en la distribución r/t.

5 En la calibración de los fechados no se restó el factor de corrección de 40 años a la edad $\mathrm{C}^{14}$.

6 El método de calibración de área de probabilidad "B " se basa en que la distribución de las edades calibradas por el método "A" de intersección con la curva no es igualmente probable.

7 Se agregó a la incertidumbre estadística de la determinación radiocarbónica (e) el error de factor de calibración (f) estimado por el LATyR en 60 años, obteniéndose un error $\mathrm{S}=\left(\mathrm{e}^{2}+\mathrm{f}^{2}\right)^{1 / 2}$ que se consignó en cada una de las agrupaciones (Grupos A, B y C).

8 Las fórmulas utilizadas para calcular la Mp y el estadístico T fueron tomadas de Figini et al. 1983: "La media ponderada ....puede ser "calibrada "..., si es necesario relacionarla con la edad histórica o astronómica " (Figini et al. 1983:53).

9 A partir de la prospección siguiendo transectas realizadas en el ambiente de fondo de valle, se localizaron, además del sitio El Carmen, 48 concentraciones de materiales de diferente densidad, y con registros cerámicos similares a los recuperados en El Carmen (Grupos cerámicos Aguada, Sanagasta, Ordinario y Negro s/Rojo). 